\title{
Statistical Prediction of Eye Locations for Drivers of Military Ground Vehicles
}

\author{
Yaser Zerehsaz (Corresponding Author) \\ Industrial and Operations Engineering Department, University of Michigan, Ann Arbor, \\ Michigan, 48109. \\ Email: yzereh@umich.edu \\ Phone: +1-734-276-5404 \\ Jionghua (Judy) Jin \\ Industrial and Operations Engineering Department, University of Michigan, Ann Arbor, \\ Michigan, 48109. \\ Email: jhjin@umich.edu \\ Phone: +1-734-763-0519
}

\section{Sheila M. Ebert}

University of Michigan Transportation Research Institute, Ann Arbor, Michigan, 48109.

Email: ebertshe@umich.edu

Phone: +1-734-615-5897

\section{Matthew P. Reed}

University of Michigan Transportation Research Institute, Ann Arbor, Michigan, 48109.

Industrial and Operations Engineering Department, University of Michigan, Ann Arbor, Michigan, 48109.

Email: mreed@umich.edu

Phone: +1-734-936-1111

\section{Acknowledgements}

This research was supported by the Automotive Research Center (ARC) at the University of Michigan, under agreement W56H2V-14-2-0001 with the US Army Tank Automotive Research, Development, and Engineering Center (TARDEC) in Warren, MI. 


\title{
Statistical Prediction of Eye Locations for Drivers of Military Ground Vehicles
}

\author{
Yaser Zerehsaz, Jionghua (Judy) Jin, Sheila M. Ebert, Matthew P. Reed
}

\begin{abstract}
In this paper, a statistical model is developed to predict the driver eye locations in military ground vehicles. The data were taken from a study on soldier driving postures and seating positions. The distribution of eye locations is represented by an "eyellipse", a geometric construction that approximates the distribution of the eye locations using an ellipse. Eyellipses have been widely used for the design of passenger cars and commercial trucks. The location and dimensions of the soldier eyellipse were developed based on the data from 145 male and female soldiers who selected their preferred driving postures in a range of vehicle layouts presented in a vehicle mockup. Driver eye locations were modeled using regression analysis. The model predicts the distribution of eye locations as a function of vehicle interior layout for a soldier population defined by the gender ratio (fraction male) and the distribution of body dimensions within each gender. This is the first eyellipse model taking into account the effects of protective equipment and body-borne gear. The model has broad applicability to the design of tactical, trucklike vehicles with fixed accelerator heel points.
\end{abstract}

Keywords: Vehicle design, eye locations, eyellipse, gear ensembles 


\section{Introduction}

Accurate prediction of driver eye locations is critical for vehicle design. Driver eye locations are used to assess direct and indirect vision outside of the vehicle and to lay out controls and displays. SAE International (formerly the Society of Automotive Engineers) developed recommended practice J941, which presents the "eyellipse” via a statistical model based on a representation of driver eye locations as a multivariate normal distribution (SAE 2016). The original passenger car eyellipse was developed in the 1960s using driver eye locations captured by stereo-photogrammetry in convertibles (Meldrum 1965). In the 1990s, Flannagan et al. (1998) developed a new version of the eyellipse that better represents the effects of vehicle geometry and driver body dimensions. An updated version of this model is now codified in J941.

A similar methodology was used by Reed (2005) to develop an eyellipse for commercial trucks and buses. Eye location data obtained in a laboratory study from 62 truck drivers were analyzed to predict the eye location as a function of steering wheel position, seat height, and driver body dimensions. The predictions corresponded well to the distributions of eye locations in six trucks driven over a road course.

No similar model is currently available for tactical military vehicles. Distinguishing characteristics of this application include the unique population and the presence of personal protective equipment (PPE), such as body armor and helmets, as well as body-borne gear including ammunition and communications gear.

The current study is based on the data from the Seated Soldier Study (Reed and Ebert 2013), which is a large-scale laboratory study of soldier postures and body shape in 
vehicle seating situations. Using these data, Zerehsaz et al. (2016) developed statistical models to predict driver-selected seat positions. These seating accommodation models can be used to ensure that soldiers have a sufficient seat adjustment range to comfortably drive vehicles. The current paper presents the methods and outputs in constructing a driver eyellipse for truck-like vehicles with a fixed heel point. The resulting eyellipse model is the first to take into account soldier body dimensions and gear ensembles.

\section{Methods}

\subsection{Construction of the Eyellipse}

Figure 1 shows an example of an eyellipse calculated for one package configuration (vehicle layout) with a midsize-male figure shown for scale. The package configuration is characterized by the height and fore-aft position of the center of the steering wheel with respect to the pedals, using dimensions H17 and L11 defined in SAE J1100 (SAE International 2016). All calculations are performed with respect to the accelerator heel point (AHP). The eyellipse has a "cutoff" characteristic illustrated schematically in Error! Reference source not found.. To be more specific, under the assumption that the underlying eye location distribution is multivariate normal (Meldrum 1965, Flannagan et al. 1998, Mannary et al. 1998), all tangents to the eyellipse (either a line in $2 \mathrm{D}$ or a plane in 3D) divides the eye location distribution into constant fractions. For example, a tangent to the commonly used " $95 \%$ eyellipse" divides the eye location distribution into $95 \%-5 \%$ fractions. (Note that the eyellipse does not enclose $95 \%$ of eye locations.) This characteristic is exploited for vision analyses where the goal is to ensure that a desired percentage of the driver population can see a particular target inside or outside the vehicle target without head movement. Figure 2 shows an up-vision analysis, 
demonstrating the maximum up-vision angle that would accommodate $95 \%$ of the driver population.

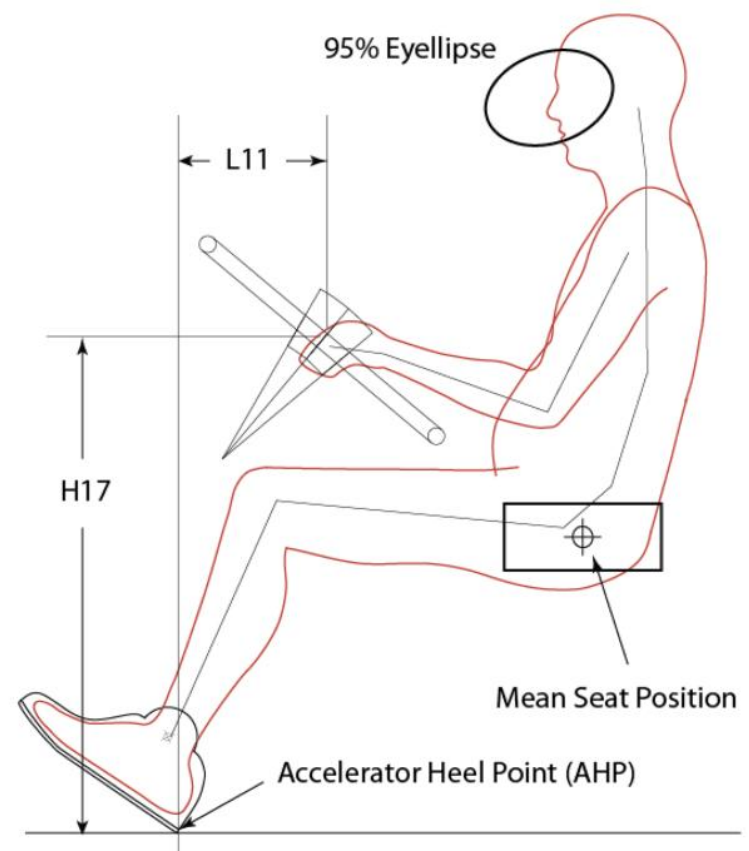

Figure 1: Example of a side-view eyellipse in the vehicle driver workspace

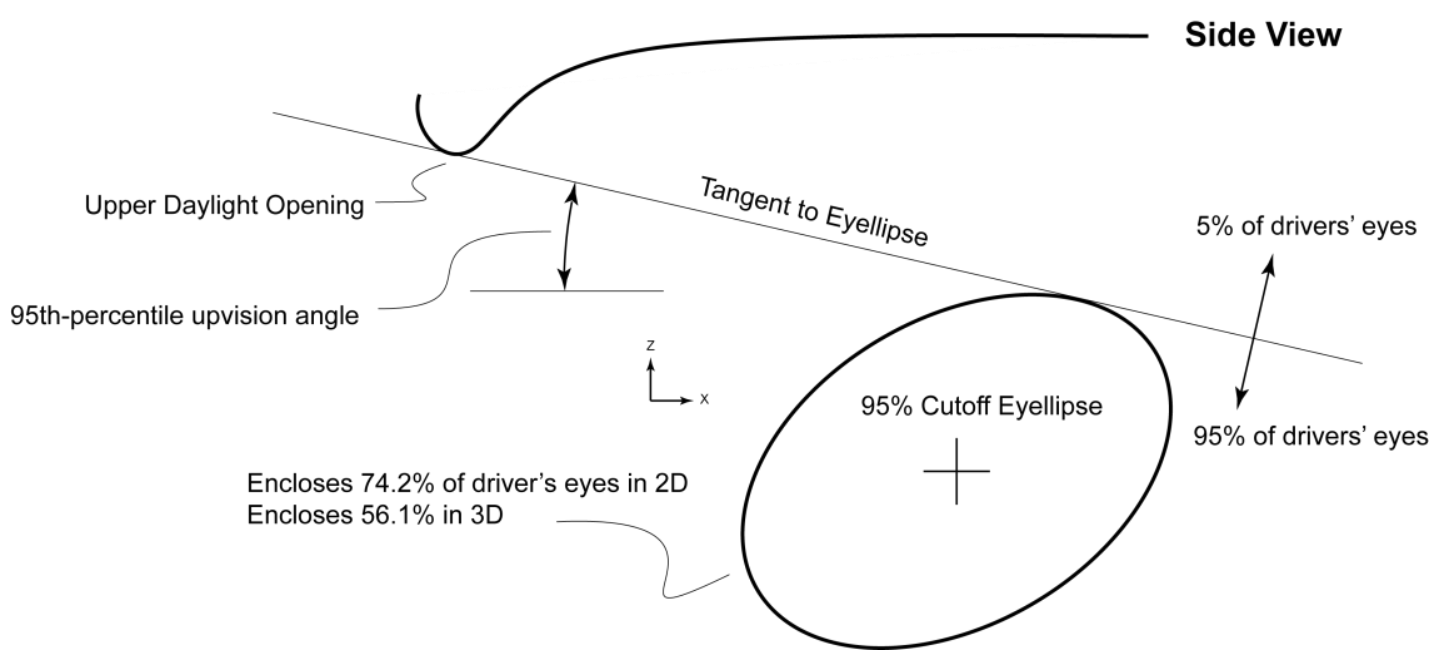

Figure 2: Illustration of side-view eyellipse with $95 \%$ cutoff characteristic 
The location and dimensions of the eyellipse are based on the statistical models described below. The model is configurable for the body dimension distributions of the driver population and gender mix. A three-dimensional normal distribution is used to represent the distribution of driver eye locations. Specifically, the data analysis suggests that the distribution of right or left eye locations for a single gender population is normal on each axis. The male and female eye location distributions overlap substantially, and conducting analyses with separate male and female eyellipses is cumbersome. Hence, a procedure is developed to create a single eyellipse approximating the whole population's cutoff behavior through weighted analyses using single-gender eyellipses.

Figure 3 shows the analysis procedure schematically. The eyellipse is inclined down at the front because shorter drivers, who also have shorter torsos, tend to sit further forward. Male and female centroids are computed along the inclined $x$ axis (the main axis of the ellipse) based on the mean stature and mean seat position for both single-gender populations, and front and rear cutoffs are computed for the combined male-female population. The vertical $(z)$ and lateral $(y)$ dimensions are computed similarly using normal-distribution approximations. The $x$ axis is the direction where the eye locations have the highest variability. The $z$ axis is perpendicular to $x$ axis and represents the direction of the second highest variation in the eye locations. In other words, $x$ and $z$ give the major and minor axes of the ellipse fitted to the eye locations in the side view, respectively. Both directions are obtained using principal component analysis (PCA). 


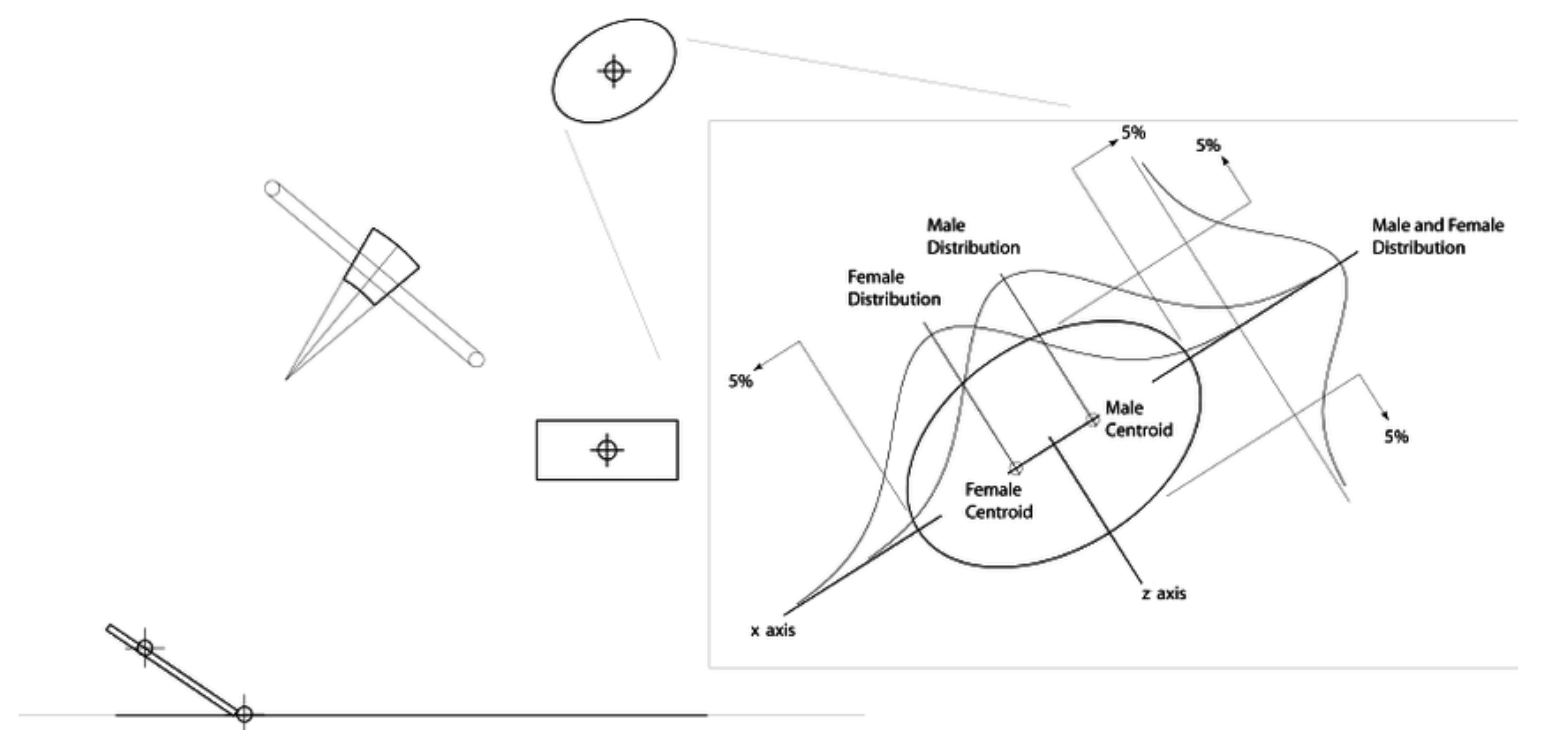

Figure 3: Schematic of eyellipse construction

\subsection{Data Source}

The data used for the current analysis were gathered in the Seated Soldier Study (Reed and Ebert 2013) through a large-scale study of soldier posture and body shape. The current analysis is based on data from 145 male and female soldiers who were measured in a reconfigurable driver mockup (Figure 4). The important variables are the eye location and the driver-selected seat position, calculated as the location of the SAE J826 H-point at the driver's chosen seat position. 


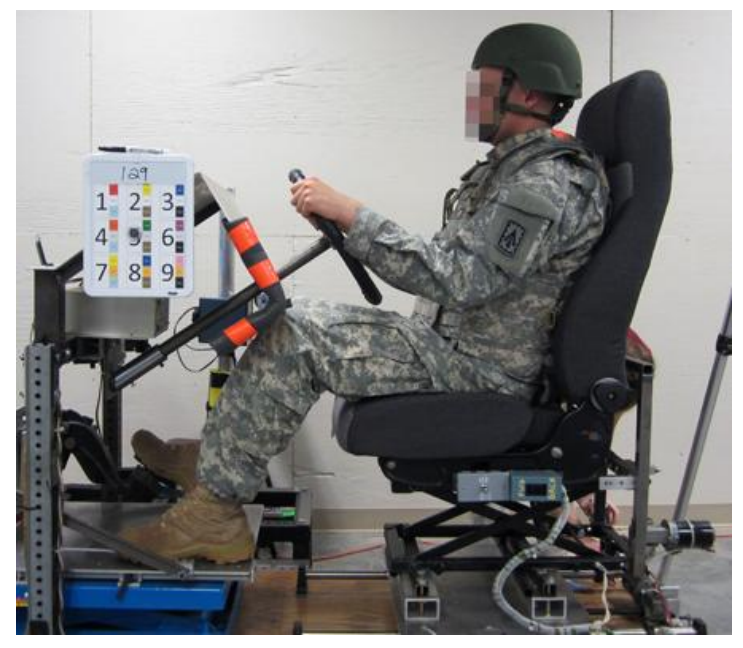

Figure 4: Driver mockup (Reed and Ebert 2013).

The summary statistics of several standard anthropometric variables are given in Table 1. Because the regression analysis presented below identified no important gender differences after considering the body size, the analyses were based on the combined male-female population information provided in Reed and Ebert 2013 (For single-gender population statistics and the distributional data for more anthropometric measures, see Reed and Ebert 2013).

Table 1: Anthropometric characteristics of the driver population (combined men and women,

\begin{tabular}{cccccc} 
& \multicolumn{7}{c}{$\mathrm{N}=145)$} \\
Variable & Mean & SD & $\mathbf{5}^{\text {th }}$ \%ile & $\mathbf{5 0}^{\text {th }} \%$ ile & $\mathbf{9 5}^{\text {th }}$ \%ile \\
\hline Stature $(\mathrm{mm})$ & 1726 & 74.2 & 1589 & 1734 & 1840 \\
\hline Body Weight $(\mathrm{kg})$ & 78.7 & 13.3 & 58.1 & 76.5 & 102.4 \\
\hline Erect Sitting Height $(\mathrm{mm})$ & 902 & 40.6 & 831 & 905 & 977 \\
\hline Sitting Height $/$ Stature & 0.523 & 0.0135 & 0.497 & 0.523 & 0.545 \\
\hline BMI $\left(\mathrm{kg} / \mathrm{m}^{2}\right)$ & 26.4 & 3.9 & 20.3 & 26.5 & 33.2 \\
\hline
\end{tabular}


The soldiers in this study were tested at three garb levels (Figure 5). The Advanced Combat Uniform (ACU) level consists of a jacket, trousers, moisture wicking shirt and combat boots. All extra items including padding on the knees and helmet or caps were removed. At the Personal Protective Equipment (PPE) level, soldiers wore an Improved Outer Tactical Vest (IOTV) with Enhanced Small Arms Protective Insert (ESAPI) plates, Enhanced Side Ballistic Inserts (ESBI), and an Advanced Combat Helmet $(\mathrm{ACH})$ in addition to the ACU ensemble. At the third level of gear, called encumbered (ENC), a hydration pack and a Tactical Assault Panel (TAP) were added to the IOTV.

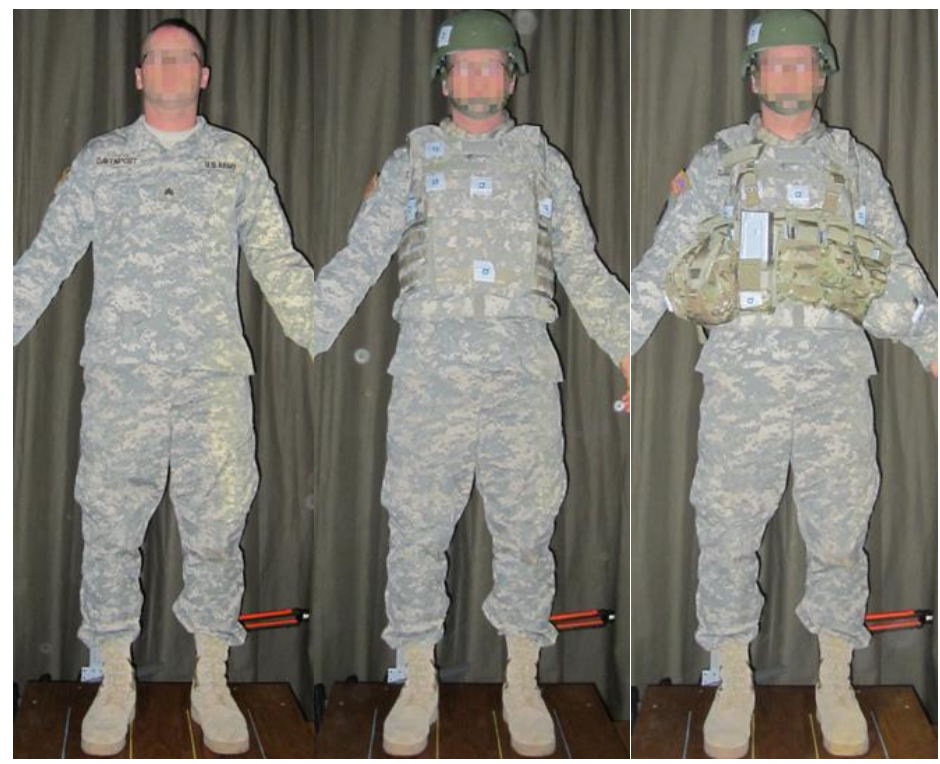

ACU PPE ENC (Rifleman)

Figure 5: ACU, PPE and ENC military garb levels

In this study, five vehicle package conditions were presented. Each condition was characterized by a different steering wheel position with respect to the pedals. In each condition, the seat was initially adjusted to the mean seating position for male soldiers, which was obtained from previous studies, and the seat back angle was set to 17 degrees, 
which is a typical design seat back angle in truck-like vehicles. All five conditions were presented in a random order at the ACU garb level and are illustrated in Table 2. To investigate the effects of PPE and ENC garb levels, Condition 5 was repeated for both additional levels.

After entering the mockup, the soldiers adjusted the fore-aft and vertical seat position and seat back angle to obtain a comfortable driving position. The soldiers' posture and seat adjustments were recorded by digitizing body and seat landmarks using a FARO Arm coordinate digitizer. The seat position was expressed as the SAE J826 Hpoint location relative to the accelerator heel point (AHP). Seat back angle was calculated based on the angle change of the seat back relative to the initial seat back angle of 17 degrees measured using the SAE J826 manikin torso angle (SAE A40).

Table 2: Driver Mockup Conditions

\begin{tabular}{cccccc}
\hline & \multicolumn{2}{c}{$\begin{array}{c}\text { Accelerator Heel Point } \\
\text { Relative to Steering Wheel }\end{array}$} & $\begin{array}{c}\text { Accelerator Pedal } \\
\text { Angle }\end{array}$ & $\begin{array}{c}\text { Initial Seat H-point } \\
\text { Relative to Steering Wheel }\end{array}$ & $\begin{array}{c}\text { Seat } \\
\text { Height }\end{array}$ \\
\hline Package $\dagger$ & $\begin{array}{c}\text { SAE L11, } \\
\text { Fore-Aft (mm) }\end{array}$ & $\begin{array}{c}\text { SAE H17, } \\
\text { Vertical (mm) }\end{array}$ & $\begin{array}{c}\text { Degrees from } \\
\text { horizontal }\end{array}$ & Fore-Aft (mm) & $\begin{array}{c}\text { SAE } \\
\text { H30 } \\
(\mathrm{mm})\end{array}$ \\
\hline 1 & 225 & 785 & 47 & 417 & 473 \\
3 & 375 & 785 & 47 & 364 & 473 \\
$5^{*}$ & 337.5 & 735 & 40 & 390 & 423 \\
7 & 300 & 685 & 33 & 417 & 373 \\
9 & 450 & 685 & 33 & 364 & 373 \\
\hline
\end{tabular}

*Repeated with PPE and ENC

$\dagger$ Conditions 2, 4, 6, and 8 were dropped after the first few sessions due to time constraints. 


\subsection{Computing the Eyellipse Location}

The eyellipse is positioned relative to the mean driver-selected seat position. The seat position model presented in Zerehsaz et al. (2016) is briefly recapped here. Table 4 shows the linear regression equations from Reed and Ebert (2013) for predicting driverselected fore-aft and vertical seat positions. The anthropometric distributions from the Army Anthropometric Survey (ANSUR) (Gordon et al. 1989) given in Table 5 are used throughout the paper when needed. The fore-aft steering wheel position L11 (see Figure 1 for an illustration of the input dimensions) is a function of vertical steering wheel position $\mathrm{H} 17$, and the relationship is given as $\mathrm{L} 11=888.75-0.75 \mathrm{H} 17$. To avoid multicollinearity of the predictors in the regression analysis, the relative fore-aft steering wheel position $\mathrm{L} 11_{\text {rel }}$ is used instead of L11. The relative L11 is computed as L11 $888.75-0.75 \mathrm{H} 17$ with values $-75,0$, and $75 \mathrm{~mm}$. The natural $\log$ of BMI is used instead of BMI to obtain a measure that is approximately normally distributed. Table 3 gives the correlation matrix of all the predictors, demonstrating a nonsignificant collinearity.

Table 3: Correlation matrix of predictors

\begin{tabular}{ccccc}
\hline Stature & $\begin{array}{c}\text { Sitting Height } \\
(\mathrm{SH}) / \text { Stature }(\mathrm{S})\end{array}$ & L11 rel & H17 & Ln(BMI) \\
\hline 1 & -0.218 & 0.001 & 0.001 & -0.034 \\
\hline-0.218 & 1 & 0.002 & 0.006 & 0.147 \\
\hline 0.001 & 0.002 & 1 & 0.018 & -0.006 \\
\hline 0.001 & 0.006 & 0.018 & 1 & 0.005 \\
\hline-0.034 & 0.147 & -0.006 & 0.005 & 1 \\
\hline
\end{tabular}


Table 4: Linear regression models for predicting fore-aft and vertical seat positions at the ACU garb level (from Reed and Ebert 2013)

\begin{tabular}{c|c|c|c|c|c|c|c|c}
\hline Mean & intercept & L11rel & H17 & Stature (S) & Ln(BMI) & $\begin{array}{c}\text { Sitting Height } \\
(\text { SH)/Stature (S) }\end{array}$ & $R_{a d j}^{2}$ & RMSE \\
\hline$\mu_{x}$ & 1014 & 0.487 & -0.780 & 0.310 & 62.2 & -923 & 0.75 & 30.3 \\
$\mu_{z}$ & -252 & -0.089 & 0.99 & -0.037 & & & 0.80 & 20.8 \\
\hline
\end{tabular}

Table 5: Anthropometric distributional parameters (mean and standard deviation) from ANSUR (Gordon et al. 2014)

\begin{tabular}{lcc}
\multicolumn{1}{c}{ Dimension (mm) } & Men & Women \\
\hline Stature (S) & $1756(68.6)$ & $1628(64.2)$ \\
Ratio of Erect Sitting Height to Stature (SH/S) & $0.523(0.0135)$ & $0.526(0.0141)$ \\
$\mathrm{Ln}(\mathrm{BMI}) \dagger$ & $3.31(0.146)$ & $3.23(0.135)$ \\
\hline
\end{tabular}

$\dagger$ Units are natural $\log \left(\mathrm{kg} / \mathrm{m}^{2}\right)$

From Zerehsaz et al. (2016), the fore-aft mean seat position can be calculated as

$$
\begin{aligned}
& \mu_{\text {seatpositionx,male }}=1278.8-0.780 \mathrm{H} 17+0.487 \mathrm{~L} 11_{\text {rel }} \\
& \mu_{\text {seatpositionx,female }}=1231.9-0.780 \mathrm{H} 17+0.487 \mathrm{~L} 11_{\text {rel }}
\end{aligned}
$$

The vertical mean seat position is estimated as

$$
\begin{aligned}
& \mu_{\text {seatpositionz,male }}=-317+0.99 \mathrm{H} 17-0.089{\mathrm{~L} 11_{\text {rel }}} \\
& \mu_{\text {seatpositionz,female }}=-312.3+0.99 \mathrm{H} 17-0.089 \mathrm{~L} 11_{\text {rel }}
\end{aligned}
$$

To simplify the application of the eyellipse model, a parsimonious model that provides a satisfactory performance is preferred over a complex model that gives only a marginally better performance. Consequently, the regression models for eye location were fitted to the data in two steps. In the first step, an automated forward stepwise regression method was utilized to find an initial set of relevant predictors using a P-value less than 0.05 to enter. In a subsequent manual process, predictors with a P-value larger than 0.01 were removed and the models were 
recomputed. The regression models for predicting the fore-aft and vertical eye locations with respect to seat $\mathrm{H}$-point are obtained as

$$
\begin{gathered}
\mu_{\text {eyeReHptx }}=345+0.116 \text { L11rel }-0.108 \text { Stature }-44 \operatorname{Ln}(\mathrm{BMI}), R_{a d j}^{2}=0.08 \\
\operatorname{RMSE}=40.1 \\
\mu_{\text {eyeReHptz }}=-816+0.411 \text { Stature }+29 \operatorname{Ln}(\mathrm{BMI})+1262 \frac{\mathrm{SH}}{\mathrm{S}}, R_{\text {adj }}^{2}=0.77 \\
\mathrm{RMSE}=17.3
\end{gathered}
$$

where $\mu_{\text {eyeReHptx }}$ and $\mu_{\text {eyeReHptz }}$ denote the fore-aft and vertical mean eye locations, respectively. Equation 5 demonstrates that fore-aft eye location relative to the seat is only weakly affected by anthropometric variables and the fore-aft steering wheel location. Not surprisingly, the vertical eye location regression has a much higher $\mathrm{R}^{2}$ value due to the effects of body size. To obtain the mean eye locations with respect to the package origin (AHP), which are called the eyellipse reference centroids, the fore-aft and vertical mean seat positions from Equations 1-4 are added to Equations (5) and (6), respectively, using the fraction of men in the driver population to establish a weighted mean.

\subsection{Computing the Eyellipse Dimensions}

The side-view angle of the eyellipse was computed by conducting a principal component analysis method to the side-view eye location data after centering the data within each condition to remove package effects. As noted above, the eyellipse is angled down at the front because shorter drivers, who also have shorter torsos, tend to sit further forward. The first eigenvector was calculated as $\mathbf{e}_{1}=(0.948,0.319)$; hence, the $x$-axis (the direction of the highest variability in the data) angle with respect to horizontal can be

computed as $\theta_{x}=\arctan \left(\frac{0.319}{0.948}\right)=18.6$ degrees to horizontal. The analysis demonstrates that this angle is not affected by vehicle configuration; as a result, the same 
angle is used in all cases. Since the lengths of the $x$ and $z$ axes are affected by the anthropometric variables, the $x$ and $z$ coordinates (principal component scores) are regressed on the drivers' attributes. The regression equations are given as

$$
\begin{gathered}
x=-564+0.327 \text { Stature, } R_{a d j}^{2}=0.23, \mathrm{RMSE}=43.8 \\
Z=-1205+0.282 \text { Stature }+1375 \frac{\mathrm{SH}}{\mathrm{S}}, R_{a d j}^{2}=0.42, \mathrm{RMSE}=28.7
\end{gathered}
$$

Both the $x$ and $z$ coordinates are significantly related to the drivers' attributes.

Consequently, the relationships in Equations (7) and (8) are used to calculate the cutoff points on each axis.

As discussed, the centroid of the eyellipse must be positioned with respect to the combined female-male mean seat position. To calculate the combined mean seat position, Equations (1) and (3) are used with the mean population values of Stature and $\frac{\mathrm{SH}}{\mathrm{S}}$. The mean population value of Stature is given by

$$
\mu_{\text {Stature,pop }}=m \mu_{\text {Stature,male }}+(1-m) \mu_{\text {Stature,female }}
$$

where $m$ is the fraction of males in the population, $\mu_{\text {Stature,male }}$ is the male's mean Stature and the female's mean Stature is represented by $\mu_{\text {Stature,female }}$. The mean population value of $\frac{\mathrm{SH}}{\mathrm{S}}$ is calculated in a similar way to that of Stature. The combined female-male eye locations with respect to H-point given in Equations (5) and (6) are estimated using the mean male and female anthropometry values. The results are added to the combined female-male mean seat position to obtain the eyellipse reference centroids. The reference centroid is used as a starting point to scale and locate the eyellipse, as follows: 
(1) Construct a side-view line passing through the eyellipse reference centroid oriented at $\theta_{x}=18.6$ degrees from horizontal (down at the front).

(2) Find the male and female centroids by moving along the $x$ line relative to the reference centroids with the distance given by

$$
\begin{gathered}
x_{\text {centroid,male }}=0.327\left(\mu_{\text {Stature }, \text { male }}-\mu_{\text {Stature }, p o p}\right) \\
x_{\text {centroid,female }}=0.327\left(\mu_{\text {Stature,female }}-\mu_{\text {Stature,pop }}\right)
\end{gathered}
$$

where the coefficient 0.327 is obtained from Equation 7.

(3) Compute the standard deviation of eye location for male and female drivers along the eyellipse $x$ axis as

$$
\begin{gathered}
\sigma_{x, \text { male }}=\sqrt{\left(0.327 \sigma_{\text {Stature }, \text { male }}\right)^{2}+(43.8)^{2}} \\
\sigma_{x, \text { female }}=\sqrt{\left(0.327 \sigma_{\text {Stature,female }}\right)^{2}+(43.8)^{2}}
\end{gathered}
$$

with the residual variance from the regression in Equation 7 is included.

(4) Choose the front axis point $x_{1}$ such that a tangent to the ellipse at that point (perpendicular to $x$ ) cuts off the desired fraction of the population $\left(F_{f}\right)$ by iteratively solving for $x_{1}$ in

$$
F_{f}=m \Phi\left(\frac{x_{1}-x_{\text {centroid,male }}}{\sigma_{x, \text { male }}}\right)+(1-m) \Phi\left(\frac{x_{1}-x_{\text {centroid,female }}}{\sigma_{x, \text { female }}}\right)
$$

where $\Phi$ is the standard normal cumulative distribution function. Similarly, choose the rear axis point $x_{2}$ to cut off the same fraction $F_{r}$ given by

$$
F_{r}=m\left(1-\Phi\left(\frac{x_{2}-x_{\text {centroid }, \text { male }}}{\sigma_{x, \text { male }}}\right)\right)+(1-m)\left(1-\Phi\left(\frac{x_{2}-x_{\text {centroid }, \text { female }}}{\sigma_{x, \text { female }}}\right)\right)
$$


The $x$ axis length is $x_{1}-x_{2}$, and the eyellipse centroid is located at $\frac{x_{1}+x_{2}}{2}$. In general, this will be slightly displaced from the reference centroid location due to the differences between men and women in the Stature variance. The length in the $z$ axis is computed similarly.

(5) Compute the male and female centroids along the $z$ axis as

$$
\begin{aligned}
& z_{\text {centroid,male }}=0.282\left(\mu_{\text {Stature,male }}-\mu_{\text {Stature }, p o p}\right)+1375\left(\mu_{\frac{S H}{S}, \text { male }}-\mu_{\frac{S H}{S}, \text { pop }}\right) \\
& z_{\text {centroid,female }}=0.282\left(\mu_{\text {Stature,female }}-\mu_{\text {Stature }, p o p}\right)+1375\left(\mu_{\frac{S H}{S}, \text { female }}-\mu_{\frac{S H}{S}, \text { pop }}\right)
\end{aligned}
$$

(6) Compute the male and female standard deviations of eye locations along the $z$ axis as

$$
\begin{gathered}
\sigma_{z, \text { male }}=\sqrt{\left(0.282 \sigma_{\text {Stature,male }}\right)^{2}+\left(1375 \sigma_{\frac{S H}{S}, \text { male }}\right)^{2}+(28.73)^{2}} \\
\sigma_{z, \text { female }}=\sqrt{\left(0.282 \sigma_{\text {Stature,female }}\right)^{2}+\left(1375 \sigma_{\frac{S H}{S}, \text { female }}\right)^{2}+(28.73)^{2}}
\end{gathered}
$$

(7) The bottom point $z_{1}$ and top point $z_{2}$ on the $z$ axis can be computed to give the bottom and top cut off fractions denoted by $F_{b}$ and $F_{t}$, respectively. The cut off fractions are calculated as

$$
\begin{gathered}
F_{b}=m \Phi\left(\frac{z_{1}-z_{\text {centroid,male }}}{\sigma_{z, \text { male }}}\right)+(1-m) \Phi\left(\frac{z_{1}-z_{\text {centroid,female }}}{\sigma_{z, \text { female }}}\right) \\
F_{t}=m\left(1-\Phi\left(\frac{z_{2}-z_{\text {centroid,male }}}{\sigma_{z, \text { male }}}\right)\right)+(1-m)\left(1-\Phi\left(\frac{z_{2}-z_{\text {centroid,female }}}{\sigma_{z, \text { female }}}\right)\right)
\end{gathered}
$$

where $\Phi$ is the standard normal cumulative distribution function. The $z$-axis length is computed as $z_{1}-z_{2}$. 
The target fraction to cut off at each end of the $x$ and $z$ axes is identical (see Figure 3) since a cut off ellipse is constructed. For example, to construct a $95 \%$ cutoff ellipse, the axis lengths are chosen to cut off $5 \%$ at each end. The eyellipse size and orientation are the same for each package, but package variables (steering wheel position) affect the centroid location. Because the eyellipse is constructed from the mean seat position, all the factors affecting the mean seat position also affect the eyellipse location.

The rear-view eyellipse can be constructed based on the lateral and vertical eye positions. Neither driver attributes nor vehicle configurations affect lateral eye location in this dataset. The mean and standard deviation of lateral eye locations with respect to AHP and driver centerline are, consequently, calculated directly from the data. The mean lateral head location is assumed to be on the driver centerline. Hence, the centroids of the left and right eyellipses are each offset from the driver centerline by half of the mean interpupillary distance of $65 \mathrm{~mm}$, i.e., $\pm 32.5 \mathrm{~mm}$ (see SAE J941). From the data, the standard deviation of lateral eye location is computed as $14.8 \mathrm{~mm}$. Since the correlation between the lateral and vertical eye locations is negligible; i.e. $\rho=0.031$ (the eyellipse is not tilted in rear view), the eyellipse dimensions in the original $\mathrm{Y}-\mathrm{Z}$ coordinates are calculated without performing PCA. The lateral axis length of the eyellipse can be computed as

$$
y=2\left(\sigma_{y} \Phi^{-1}\left(F_{y}\right)+\mu_{y}\right)
$$

where $F_{y}$ is the desired cutoff fraction (e.g., 0.95), $\Phi$ is the cumulative standard normal distribution, mean lateral eye location with respect to driver centerline is 
$\mu_{y}= \pm 32.5 \mathrm{~mm}$, the lateral eye location standard deviation is $\sigma_{y}=14.8$, and the lateral axis length is $y$.

\subsection{Effects of Garb Level}

The addition of PPE and equipment shifts the occupant forward relative to the seat. Using the results reported in Reed and Ebert (2013), PPE shifts the mean eye locations with respect to $\mathrm{H}$-point forward by $56.3 \mathrm{~mm}$ and upward by $13 \mathrm{~mm}$ relative to the ACU conditions described by Equations 5 and 6. The mean seat positions are shifted rearward by $20.8 \mathrm{~mm}$ and downward by $2.5 \mathrm{~mm}$. As a result, the reference centroids are adjusted forward by $35.5 \mathrm{~mm}$ and upward by $10.5 \mathrm{~mm}$ for the PPE condition.

When soldiers wear ENC garb level, the mean eye location is shifted forward relative to the seat $\mathrm{H}$-point by $103 \mathrm{~mm}$ and upward by $13 \mathrm{~mm}$ compared to the ACU level. The mean seat position is shifted $64.7 \mathrm{~mm}$ rearward and $7.5 \mathrm{~mm}$ downward. This leads to the reference centroids shifting $32.5 \mathrm{~mm}$ forward and a $5.5 \mathrm{~mm}$ upward relative to ACU.

\subsection{Results}

The Appendix lists the numerical results of sample calculations. Figure 6 shows $95 \%$ eyellipses for the five package configurations used in the Seated Soldier Study. In general, the eye locations follow the steering wheel (higher steering wheel produces higher eye locations) with some lag. 


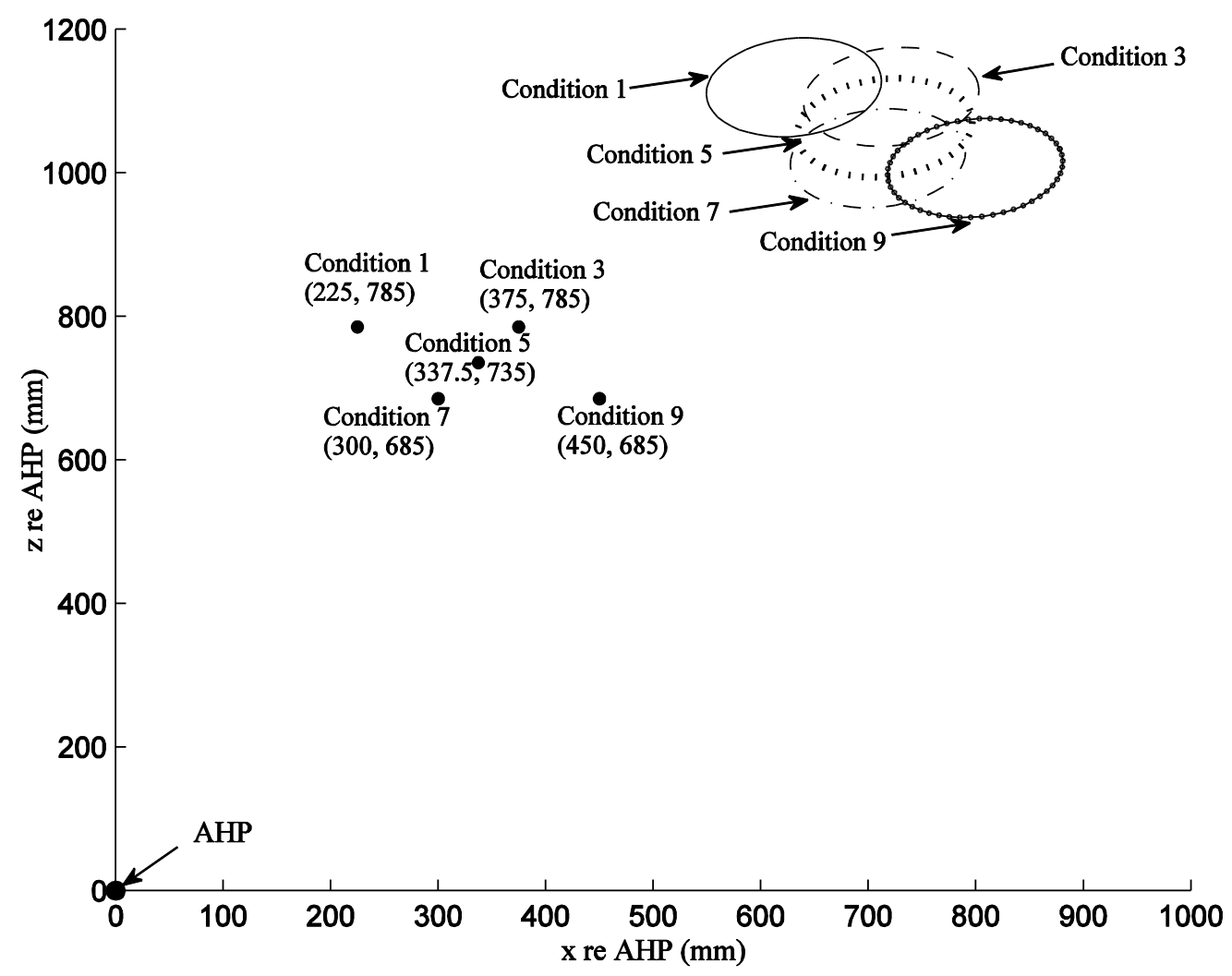

Figure 6: Effects of steering wheel location on driver eyellipse for the test conditions in Table 1

Figure 7 shows examples of a 95\% cutoff eyellipse for all three garb levels at a single vehicle package. In general, drivers' eyes are further forward and slightly higher when they are wearing body armor, but the addition of the rifleman kit (ENC) changes the position only slightly. 


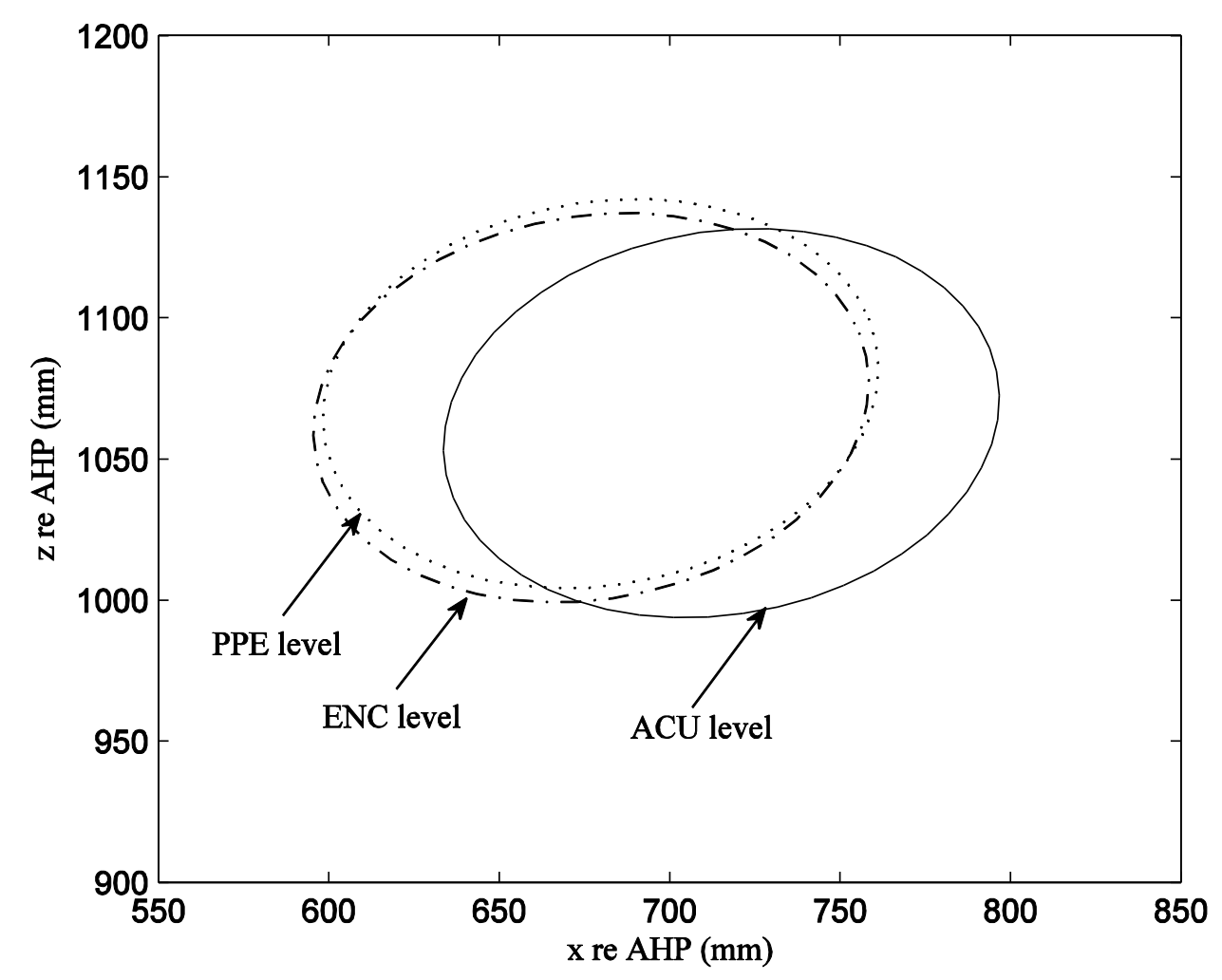

\section{Discussion}

Figure 7: Effects of garb level on the eyellipse

This paper presents the first statistical description of soldier eye locations in truck-like tactical vehicles. The model is also the first eyellipse for any application to take into account the effects of personal protective equipment and body-borne gear. The model is parametric for population attributes and can be readily adapted to a wide range of populations.

The eyellipse can be applied in the same manner as previously published eyellipses (Meldrum 1965; Flannagan et al. 1998; Reed 2006). SAE J941 and J1052 describe some standardized methodologies for the use of eyellipses in vision analysis (SAE International 2016). In general, tangents to the eyellipse (or planes in 3D) divided 
the predicted driver eye location distribution into fractions based on the target eyellipse fraction (e.g., 95\%). These constructions can be used to ensure that the desired percentage of the driving population has a particular upvision angle out of the cab, can see a prescribed target in front of the vehicle, or has a sufficient field of view in a mirror (Roe 1993).

The primary limitation of this work is that no in-vehicle validation data are available. In a previous study of commercial truck drivers, driving postures in a laboratory mockup were found to be a good predictor of in-vehicle postures (Reed 2005), but validation of the current model would be valuable. Nonetheless, the eyellipse presented here provides the first published model of soldier eye locations as drivers and represents a substantial improvement over existing design practice for military vehicles.

Further work is needed to extend these results to other vehicle scenarios, such as those with much lower seat heights. Different control layouts (for example, a joystick rather than steering wheel) and alternative external vision systems (driving via a video display rather than direct vision) may also alter drivers' posture-selection behaviors and their eye locations.

The model presented here does not take into account the censoring effect of driver posture due to cab geometry restrictions. For example, if the driver's head location is restricted by a low roof, the distribution of eye locations is likely to be shifted downward. Additional research will be needed to quantify those effects.

The eyellipse model presented above is based on the seat H-point measured using the SAE J826 H-point manikin and procedures. Recent research has shown that the ISO 
5353 Seat Index Point Tool (SIPT) can be used as an alternative measurement tool if the J826 manikin is not available (Reed and Ebert 2014). Across a wide range of seating conditions, the seat index point (SIP) was on average $5 \mathrm{~mm}$ rearward of the J826 H-point. Consequently, an estimated H-point for use with these models can be generated by translating the SIP forward by $5 \mathrm{~mm}$ relative to the seat.

Although the current model is relevant to a particular category of vehicles, with an associated population, the modeling methodology is generic and applicable to the cases when the seated operator workstation produces a range of eye locations that need to be taken into account in the design. The functional modeling approach used in this work can be applied to aviation, industrial workstations, and off-highway equipment, to name a few areas. The primary input is an appropriately parameterized dataset from a relevant population.

\section{ACKNOWLEDGMENTS}

This research was supported by the Automotive Research Center (ARC) at the University of Michigan under the agreement W56H2V-14-2-0001 with the US Army Tank Automotive Research, Development, and Engineering Center (TARDEC) in Warren, MI. We thank Gale Zielinski and Frank Huston of TARDEC for their assistance and support for the project, including their careful review of the models and documentation presented in this paper. 


\section{REFERENCES}

Flannagan, C.A.C., Manary, M.A., Schneider, L.W., and Reed, M.P. (1998). An Improved seating accommodation model with application to different user populations. SAE Transactions: Journal of Passenger Cars, 107(6): 1189-1197.

Gordon CC, Churchill T, Clauser CE, Bradtmiller B, McConville JT, Tebbetts I, and Walker RA (1989) 1988 Anthropometric Survey of U.S. Army Personnel: Methods and Summary Statistics. NATICK/TR-89/044. U.S. Army Natick Soldier Research, Development, and Engineering Center, Natick, MA.

Meldrum, J.F. (1965). Automobile driver eye position. Technical Paper 650464.

Warrendale, PA: Society of Automotive Engineers, Inc.

Manary, M.A., Flannagan, C.A.C., Reed, M.P., and Schneider, L.W. (1998).

Development of an improved driver eye position model. SAE Transactions: Journal of Passenger Cars, 107(6): 43-50.

Reed, M.P. (2005). Development of a New Eyellipse and Seating Accommodation Model for Trucks and Buses. Technical Report UMTRI-2005-30. University of Michigan Transportation Research Institute, Ann Arbor, MI.

Reed, M.P. and Ebert, S.M. (2013). The Seated Soldier Study: Posture and Body Shape in Vehicle Seats. Technical Report 2013-13. University of Michigan Transportation Research Institute, Ann Arbor, MI. 
Reed, M.P., and Ebert, S.M. (2014). Evaluation of the Seat Index Point Tool for Military Seats. Technical Report UMTRI-2014-33. University of Michigan Transportation Research Institute, Ann Arbor, MI.

Roe, R. (1993). Occupant Packaging. In Peacock and Karwowski, eds. Automotive Ergonomics. Taylor and Francis.

SAE International (2016). SAE Handbook. Warrendale, PA: Society of Automotive Engineers, Inc.

Zerehsaz, Y., Jin, J., Ebert, S.M., and Reed, M.P. (2016). Development of seating accommodation models for soldiers in vehicles. Ergonomics, DOI:10.1080/00140139.2016.1184760 


\section{APPENDIX}

\section{EXAMPLE CALCULATIONS}

All dimensions are in mm unless otherwise noted

ANSUR II (2013)

\begin{tabular}{c|cc|cc}
\hline Dimension & \multicolumn{2}{|c|}{ Men } & \multicolumn{2}{c}{ Women } \\
\hline & Mean & SD & Mean & SD \\
\hline Stature (S) & 1756 & 68.6 & 1628 & 64.2 \\
\hline Erect Sitting Height (SH) & 918 & 35.7 & 857 & 33.1 \\
\hline Stature minus Sitting Height (SSH) & 837 & 46.5 & 772 & 44.4 \\
\hline$\frac{\text { SH }}{\mathrm{S}}$ & 0.523 & 0.0135 & 0.526 & 0.0141 \\
\hline $\operatorname{Ln}(\mathrm{BMI})^{*}$ & 3.31 & 0.146 & 3.23 & 0.135 \\
\hline
\end{tabular}

*Note -- this is natural log of BMI

Fraction Male

0.9

\begin{tabular}{|c|c|c|}
\hline \multicolumn{3}{|c|}{ Vehicle Geometry } \\
\hline SWX (L11) & 337.5 & $\mathrm{~mm}$ \\
\hline SWZ (H17) & 735 & $\mathrm{~mm}$ \\
\hline L11rel & 0 & $\mathrm{~mm}$ \\
\hline A40 & 0 & $\mathrm{~mm}$ \\
\hline H30 & 423 & $\mathrm{~mm}$ \\
\hline Ensemble Level & PPE & ACU, PPE, ENC \\
\hline Hydration Pack Relief & No & Yes, No \\
\hline Calibration Tool & J826 & J826, SIPT \\
\hline
\end{tabular}




\begin{tabular}{|c|c|c|c|}
\hline \multicolumn{4}{|c|}{ Eyellipse } \\
\hline Eyellipse centroids & $\mathrm{X}$ & $\mathrm{Y}$ & $\mathrm{Z}$ \\
\hline Right & 678.9 & 32.5 & 1078.1 \\
\hline Left & 678.9 & -32.5 & 1078.1 \\
\hline
\end{tabular}

\begin{tabular}{lccc}
\hline \multicolumn{3}{c}{ Side-view eyellipse } \\
\hline Eyellipse angle $\left(\theta_{x}\right)$ & 18.6 & $\operatorname{deg}$ \\
\hline Axis length $(x)$ & 166.9 & $\mathrm{~mm}$ \\
\hline Axis length $(z)$ & 136 & $\mathrm{~mm}$ \\
\hline
\end{tabular}

\section{Rear-view eyellipse}

Axis Length (y)

Axis Length (z)
48.7

136.8 $\mathrm{mm}$

$\mathrm{mm}$ 